\title{
Mechanisms to Engage an Online Community in Crowdsourcing: Insights from an Idea Contest in Training
}

\author{
ADRIANO SOLIDORO \\ adriano.solidoro@unimib.it \\ University of Milano-Bicocca
}

FRANCESCO ALEOTTI

francesco.aleotti@unimib.it

University of Milano-Bicocca

\section{GIANLUIGI VISCUSI}

g.viscusi@imperial.ac.uk

Imperial College London

CHRISTOPHER L. TUCCI

c.tucci@imperial.ac.uk

Imperial College London

DAVIDE DIAMANTINI

davide.diamantini@unimib.it

University of Milano-Bicocca

\begin{abstract}
Knowledge sharing is particularly important for co-creating, discussing, or acquiring innovative ideas. Crowdsourcing, as an enabler of open innovation, has raised the question about the kind of organising forms and/or managerial interventions it may require or underpin. However, there is little consensus in management studies on how to best design a crowdsourcing initiative (contest) with regard to the mechanisms to engage an online community. In this paper, starting from an exploratory case study on the project "Stati Generali della Formazione e del Lavoro" (General Assembly on Training and Work)-a crowdsourcing experience designed for a large community of professional trainers, planned and managed by University of Milano-Bicocca and AIF Academy (Associazione Italiana Formatori), a broad representative association of Italian trainers-we study the factors influencing the decision of the participants (a.k.a., solvers) to become involved (and to what extent) in a contest. The study could contribute to the debate on crowdsourcing by both
\end{abstract}


underlining important governance factors involved and providing empirical evidence of the link between management strategies and crowdsourcing success.

\section{Introduction and motivation}

Innovation contests have been used by an increasing number of organisations for knowledge sharing and for co-creating or acquiring innovative ideas with the help of the crowd. However, although there are a growing number of studies on how innovation and crowdsourcing should be managed for commercial as well as societal purposes (Stieger et al., 2012; Majchrzak and Malhotra, 2013, 2020; Malhotra and Majchrzak, 2014), in management studies there is still little consensus on how to best design and implement a contest in terms of the mechanisms to engage, strengthen and monitor an online community. Since the success of a contest depends on different factors, such as the number of participants, their level of engagement as well as the quality of their contributions, this paper investigates the issues related to the design of a contest and the management strategy to facilitate it. For example, sharing introductory knowledge in order to help the participants to better comprehend the aim and nature of the contest and of the different challenges; revealing the identity and status of the content sponsors; using members of staff as contributors to "seed" the contest in the early stages; gamifying the contest assigning scores and prizes, and so on.

In the case observed, these kinds of interventions seemed more likely to facilitate participants' experiences and support a sense-making process. Therefore, this paper may help define guidelines for the purpose of maximising results of contest design.

Our core argument is that contest proponents or organisers of the contest event ("seekers") can reduce several factors of uncertainty, and therefore may influence participants' decisions to get involved via different strategies. In particular, in this paper, we study three factors influencing the decisions of the participants ("solvers") to become involved (and to what extent) in a contest: (1) users' level of contribution; (2) user behaviour; and (3) the quality of user contributions.

The paper is structured as follows. First, we discuss the theoretical background of the research, especially focusing on open innovation and crowdsourcing. Then, we outline the method adopted for the study before presenting the main results and their discussion. Finally, we offer conclusive remarks and end the paper outlining future work.

\section{Theoretical background}

The research on Open Innovation defined as "a distributed innovation process based on purposively managed knowledge flows across organizational boundaries" (Chesbrough and Bogers, 2014: 27) has moved from a firm-centric perspective to other innovation phenomena not necessarily tied to it (Bogers et al., 2017: 9). Accordingly, Open Innovation research includes the study of Knowledge Collaboration in Online Communities (Faraj, Jarvenpaa and Majchrzak, 2011; Faraj et al., 2016) and practices such as Crowdsourcing (Leimeister et al., 2009; Afuah and Tucci, 2012; Boudreau and Lakhani, 2013; Tucci, Afuah and Viscusi, 2018) oriented to engage a collective intelligence (Malone and Bernstein, 2015; Malone, 2018) in finding solutions or new ideas for innovation problems (Blohm et al., 2011) or policymaking and participatory and deliberative processes (Aitamurto and Landemore, 2016). 
However, both online communities and crowdsourcing as ways of doing Open Innovation have raised the question about the organising forms they eventually require or underpin, as well as their novelty, if any (Buganza and Verganti, 2009; Siobhan, 2011; Puranam, Alexy, and Reitzig, 2014; Biancani, McFarland and Dahlander, 2014; Dahlander and Piezunka, 2014; Piezunka and Dahlander, 2018; Linus, Jeppesen, and Piezunka, 2019). In addition, the crowd in "crowdsourcing" has been considered a subject of investigation for moving from talking about "openness broadly, in the abstract" to "a more fine-grained, analytic nuance that is needed to specify when certain forms or governance structures make sense, and when not - and what an organization should be open to and why", as pointed out by Felin et al. (2015: 132).

\subsection{Crowd dynamics and organising}

Several articles have investigated how open innovation can foster knowledge sharing (Buganza and Verganti, 2009) for example, through online communities (Fleming and Waguespack, 2007; Faraj et al., 2011; Majchrzak, Jarvenpaa and Bagherzadeh, 2015). However, the 'fluidity' (Faraj et al., 2011) regarding the traditional characteristics of a community have raised questions on the dynamics that collective forms of collaboration may enact through open innovation. Moreover, those dynamics have been empirically observed, for example, by Marchegiani et al. (2020), who analyse initiatives set by a public administration for stimulating collaboration and knowledge sharing through business social networks (BSN) of, for instance, small and medium enterprises (SMEs).

Consequently, a stream of research has concentrated on understanding the different dynamics that can lead from groups to online communities, or to open rather than closed crowds in crowdsourcing challenges and idea competitions, that is, contests (Seidel, Langner and Sims, 2016; Cross et al., 2017; Viscusi and Tucci, 2018; West and Sims, 2018; Sims and Woodard, 2019). Moreover, the study of these dynamics in crowd-based problem solving has focused attention on the factors having an impact on team performance in crowdsourcing. Accordingly, these contributions connect the research on organising forms in crowdsourcing to the stream of studies that investigate coordination in temporary groups (Valentine and Edmondson, 2014) or the management and assembly of experts from the crowd (Retelny et al., 2014; Valentine et al., 2017). Moreover, a stream of research relevant to our study is the one interested in collaboration processes and team performance in crowdsourcing environments with the aim of identifying the appropriate design of online environments for increasing the level of performance. For example, Riedl and Woolley (2016: 397) have analysed the mediation of some factors such as temporal burstiness of activity and information diversity on the effect of skill and monetary incentives on team performance in crowdsourcing.

Taking these issues into account, the analysis of Crowd Dynamics in challenges or idea competitions has been considered as relevant to identifying how the different configurations (crowds, communities, groups) that may emerge from the activities and interactions of the "solvers" may lower (or increase) "Crowd Capital". Early conceptualised by Prpic and Shukla (2013) and Prpić et al. (2015), we define "Crowd Capital" as the total number of crowd units having a demonstrated effectiveness in idea generation or task achievement (Tucci, Viscusi and Gasparetto, 2016), where it is worth noting that the terms refer not necessarily only to the winning ideas but also to the other propositions and capabilities that can be forgotten or lost in the different phases of a crowdsourcing initiative. 


\subsection{Engagement in crowdsourcing}

Engagement generally refers to involvement or commitment and, although having been firstly considered as a construct related to employee motivation and organisational performance, over the years it has also been analysed in multiple contexts and by different approaches (Schaufeli, 2014). In this article, we are specifically interested in some specific instances of the concept of "engagement". On one hand, we consider the engagement of the user when using technology (user engagement), defined as "a category of user experience characterized by attributes of challenge, positive affect, endurability, aesthetic and sensory appeal, attention, feedback, variety/novelty, interactivity, and perceived user control" (O'Brien and Toms, 2008: 941); on the other hand, we derive from science, technology and society (STS) studies the notion of citizen engagement for participation in public consultations where "information is conveyed from members of the public to the sponsors of the initiative, following a process initiated by the sponsor" (Rowe and Frewer, 2005: 255).

Taking these issues into account, Open Innovation communities have been investigated as to their relationship with emergent mechanisms of leadership and engagement, especially emphasising the role of trust, hierarchies, and the specific relevance of boundary spanners and brokers to reinforce and unify the community itself (Fleming and Waguespack, 2007). When adopting specific technological platforms - within or across different organisations - for Open Innovation initiatives, the dimension of engagement shows the relevance of some factors observed in the process of adoption of social media at the company level, such as the influence of organisational culture and the style of leadership (Parry and Solidoro, 2013). However, a different perspective emerges when considering crowdsourcing: the state-of-the-art literature has identified different issues worth taking into account when designing challenges and contests in order to support active participation and engagement; and these span from a focus on characteristics-such as task specificity, degree of idea elaboration, organisational appearance, timeline, incentives, and target group - to motivations related to learning, direct compensation, self-marketing, and social motives (Leimeister et al. 2009: 202-206). Although these are factors general enough to be applied (hypothetically) to any crowdsourcing initiative, it is worth noting that most of the literature so far has focused attention on the private sector; thus, in this paper, we aim to contribute to the research on engagement in crowdsourcing by considering the specific patterns, mechanisms, and motivations ( $\mathrm{O}^{\prime}$ Brien and Toms, 2008) that may emerge in conjunction with or autonomously to the engagement of the different actors involved in crowdsourcing initiatives developed in the public sector with the aim of policy or social innovation.

Fuger et al. (2017) have carried out an exploratory case study on communities engaged in crowdsourcing initiatives for social innovation in developing countries in order to investigate the differences between teams and individual community members and identify the distinct levels of collaboration as well as the different roles in contributing to the initiatives and the impact on team performance. Furthermore, Bonazzi et al. (2017) have proposed a model for the assessment of the cost and effectiveness of the intersection between crowd and experts for scenario planning in the public sector.

Thus, our contribution is a more fine-grained understanding of crowd engagement practices. Considering the framework proposed by Storbacka et al. (2016), we observe how actors' dispositions (i.e., initial motivations) relate to engagement, the configuration of engagement properties (e.g., relational, informational, and temporal properties), the type of platform 
provision, and its eventual engagement-related network effects (i.e., the mechanisms of supporting the engagement of participants).

\section{Method}

This research project represents an exploratory case study (Yin, 2009), where we adopt abductive reasoning (Bamberger, 2018) to the empirical exploration of patterns emerging from the corpus of data. In particular, an exploratory abduction-based approach was chosen as the primary mechanism to proceed, considering that we are studying a context that has specific characteristics: a contest involving a community of practitioners organised by a professional association together with a university department for both education and policy goals. The abductive approach is due to the specific research subject and domain where we are "confronted with puzzling facts, but unable to cleanly apply a theory or theoretical perspective to readily explain them" (Bamberger, 2018: 4); therefore, we use the "pattern of results to conceive a plausible explanation" (Bamberger, 2018: 4). Furthermore, abduction allows us to move from the observation to the identification of patterns and the generation of plausible explanations for the emergent organising practices of engagement in the specific domain of a crowdsourcing initiative (contest) for practitioners in training, where extant state-of-the-art theories (such as uncertain reduction theory) are considered as assumptions to be challenged against the observation data or as post-hoc explanations (Bamberger, 2018; Robinson, 2019).

Taking these issues into account, we have built a unique dataset that includes both quantitative and qualitative data (the text of the comments and interactions among the participants). These latter data have been analysed through a qualitative research approach for eliciting how different actors produce an account of their situated actions and make sense of their participation in the contest as members of a crowd, of a community, or a specific group to carry out the contest as an "organized artful practice" (Garfinkel, 1967: 11). Accordingly, considering the focus on the emergent organising practices rather than on the organisation promoting the challenges, in this article we adopt an ethnomethodological perspective (Garfinkel, 1967) as a complement to abductive reasoning guiding the triangulation of data and methods (Jick, 1979; Olsen, 2004).

For the data collection and analysis, three of the authors were directly involved in the development of the challenges and acted as observers as well as collectors of the data through the crowdsourcing online platform making up the final data set together with personal memos and reflections. The corpus of data has been subsequently collaboratively analysed by all the authors or subset of them from March to June 2020 through a series of Zoom sessions of 30 minutes average (approximately one session/month), where for any session at least one of the two authors not directly involved in the challenges acted as critical external discussant. The goal was to understand whether and how (through which procedures, patterns, or organising forms) a social order is eventually produced in crowdsourcing and how the different actors involved account for it.

\section{The study}

The digital platform Crowdicity (Unimib.crowdicity.com, 2020) hosted from June 2019 to December 2019 the project "Stati Generali della Formazione e del Lavoro" (General Assembly 
on Training and Work), a crowdsourcing experience designed for a large community of professional trainers, planned and managed by University of Milano-Bicocca and AIF Academy (Associazione Italiana Formatori), a broad representative association of Italian professional trainers. The final goal of the initiative was the drafting of a wiki report or community-based report and guidelines for policymaking: the "Libro Bianco della Formazione e del Lavoro", a White Book to be eventually presented to the Minister of Labor in which the community of Italian trainers summarise the collective re-elaboration and analysis of the present changes in society and in the workplace context and outline strategies and guidelines for the future development of the training sector in Italy.

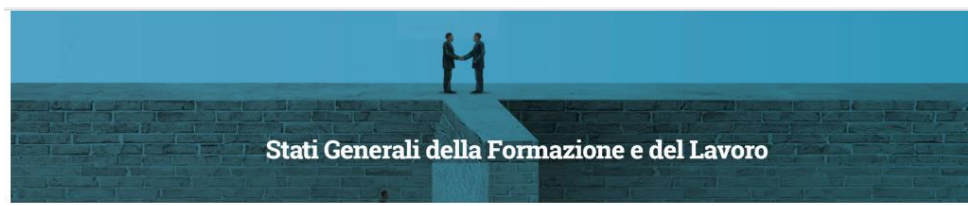

Sfide

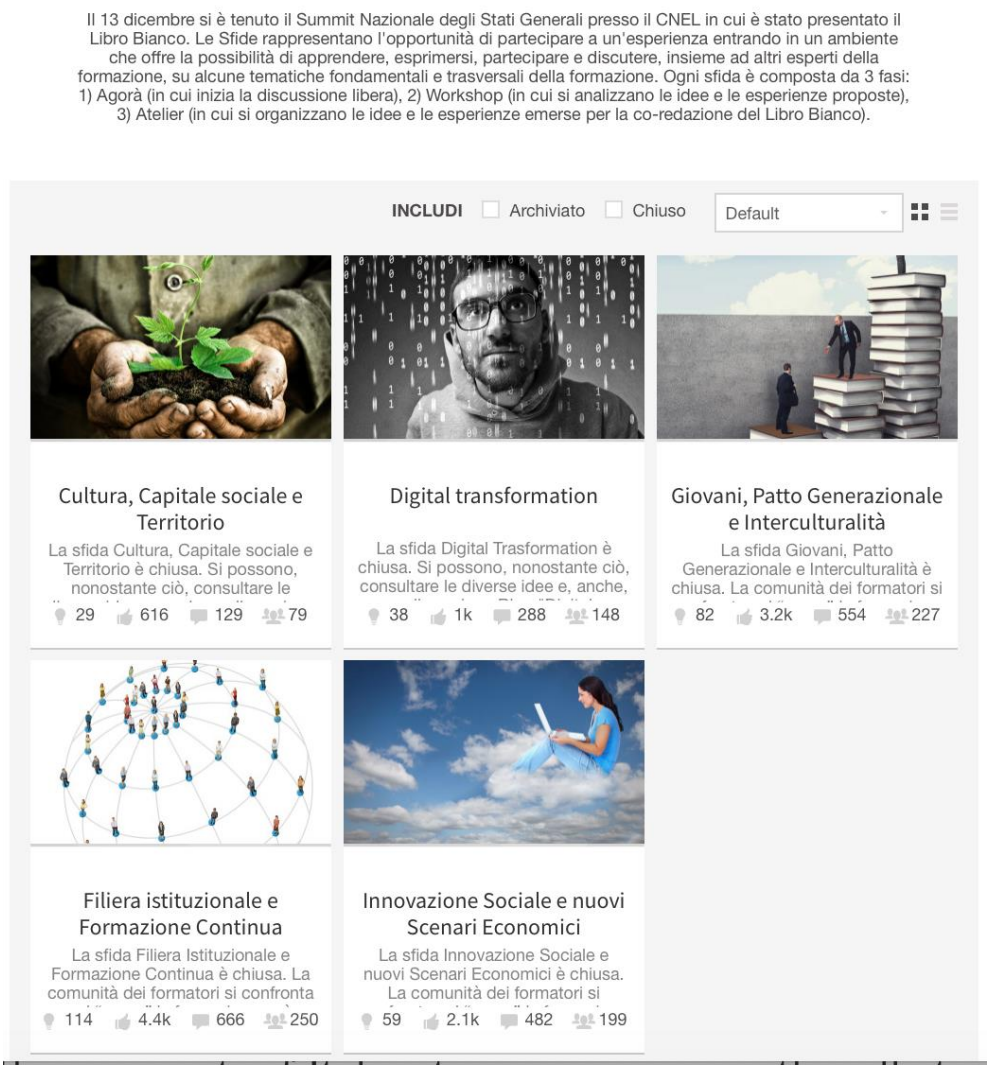

Figure 4.1. The interface of the crowdsourcing platform proposing the five competitive categories ("challenges")

The contest proposed five competitive categories ("challenges", in Figure 4.1) around five macro-themes related to changes in society and in the professional environment and, therefore, as well as to the sector of vocational education and training: (1) culture, social capital and territory; (2) digital transformation; (3) social innovation and new economies; (4) young people, intergenerational relationships and multiculturalism; and (5) institutional value chain and life-long 
training. Introductory content was provided to help the participants to better understand the nature of the challenges and reach a basic common knowledge.

\subsection{Roles and structure of the challenge}

Participants were asked to post innovative ideas and solutions about the issues illustrated in each one of the five challenges, and therefore discuss and comment on other users' opinions and eventually vote on the best solutions. Staff members actively contributed as facilitators supporting and stimulating the discussion on the platform. Moreover, activities and contributions of the participants were supervised by an editorial board that evaluated their acceptability in terms of pertinence and relevance.

In Italy, trainers and vocational educators are required to attend professional courses offered annually by certified agencies that are authorised to provide the qualifications needed for the profession. AIF Academy is one of the agencies providing the qualification, and for this reason trainers who took part at the "Stati Generali" project were able to apply for their qualification/certificate. To quantify the level of engagement on the participants, it was decided to assign a score to the different activities each user would perform on the platform: post an idea, comment on other ideas, and vote for preferences. Upon reaching a predetermined score, the qualification was assigned to the participants. The three authors involved as observers could detect that the motivation of the crowd was linked both to the need to acquire a qualification, and to the interest in an uncommon professional experience in the form of knowledge sharing and social learning.

Each challenge remained open for approximately five weeks and was structured in different phases: (1) Pre-start (some introductory content was uploaded onto the crowdsourcing platform by the editorial board); (2) Agora (ideas were proposed by the participants and put on display); (3) Atelier (most voted ideas were selected, discussed, and eventually those judged more interesting by the crowd were selected and refined through a co-creation session; (4) The challenge was closed (no more ideas or comments could be uploaded); (5) Follow-up (ideas and discussions content were edited in order to be included in the community-based report/White Book).

\subsection{Trend of contributions}

The number of users who subscribed to the platform continued to increase gradually until the closure of the project (end of December 2019), when their total number reached 261. For the duration of the crowdsourcing event, the staff's activity was fundamental: 20 staff members-called "facilitators" - actively contributed by supporting and stimulating the discussion on the platform. 
Users (261) and facilitators (20) contributions (ideas+comments) and relative trends

70

50

50

40

30

20

10

0 …...

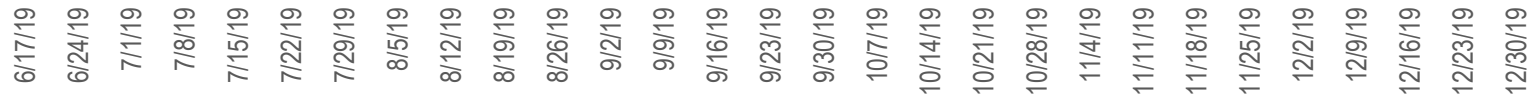
users facilitators Lineare (users) Lineare (facilitators)

Figure 4.2.1. Trend of contributions by users and facilitators.

Looking at the trend of users' activity on the platform (ideas and comments posted), we observed an initial period of two months (June and July) with few contributions posted (this was also due to the lower number of users who had subscribed to the platform compared to those in the following months. From September 2019 onwards, however, activity was much more intense, with peaks of about 60 contributions per day. As shown in Figure 4.2.1, starting from September, users' contribution increased, while the facilitators' remained stable for the entire duration of the online event.

Moreover, we have highlighted the presence of an incubation period, that is, this time frame was necessary for users to learn how the platform worked and how to carry out the assigned tasks, as well as to get familiar with the conceptual framework of each challenge and therefore-in terms of interaction within the crowd - to reach a sufficient level of contributions to fuel the discussion. During this incubation period, the number of ideas and comments coming from the facilitators sometimes exceeded those of the users. After some weeks, however, the discussion within the community was self-sustaining and the role of the staff became more marginal. Figure 4.2.2 shows the ratio between facilitators' and users' contribution in each challenge. Looking at the trends, we can spot an increase of users' contributions challenge after challenge (which were distributed in a temporal sequence), as well as a decrease of intervention by the facilitators, until the ratio reached a stable equilibrium (see for example, Challenge 5 in Figure 4.2.2). 


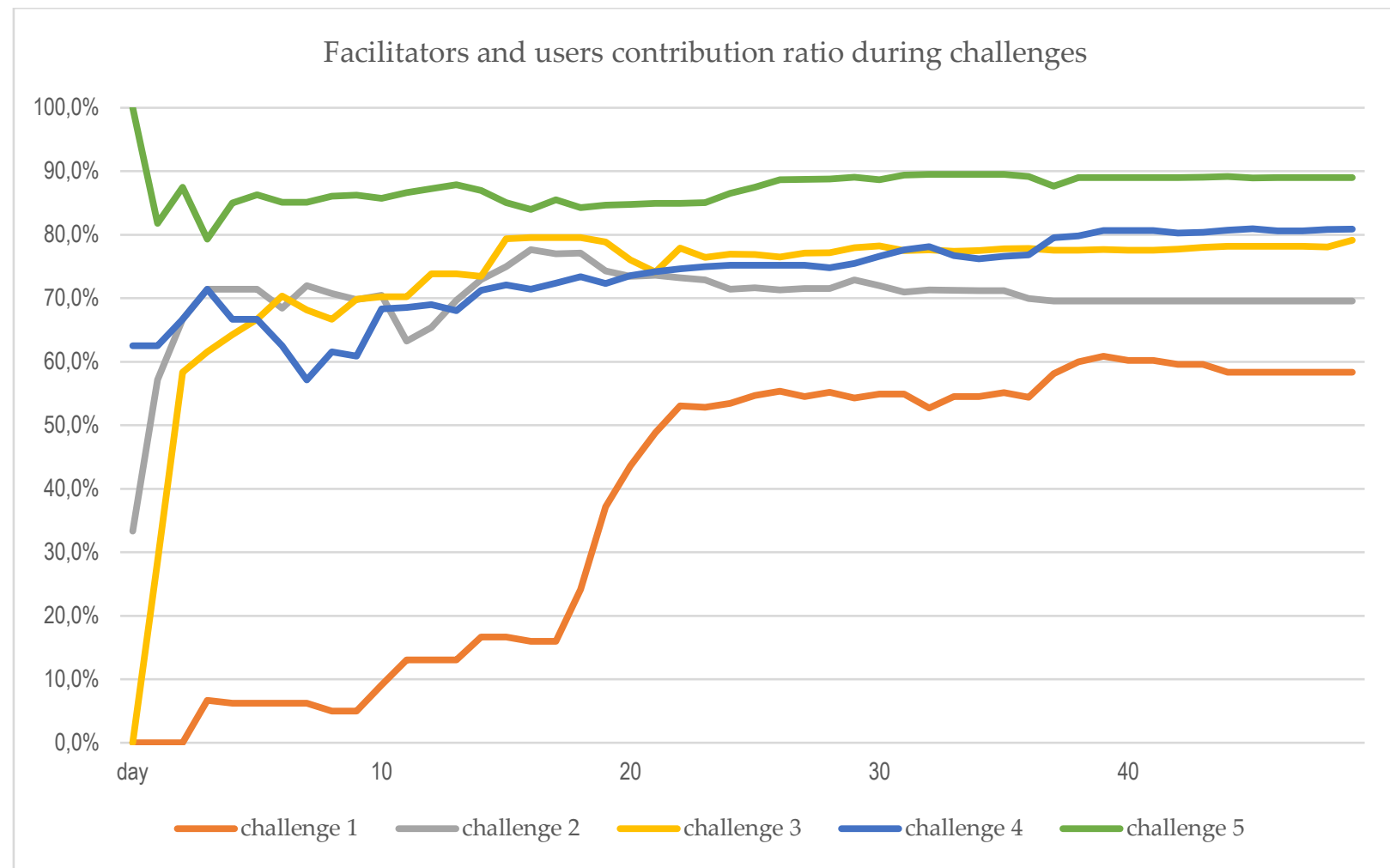

Figure 4.2.2. Ratio between users' and facilitators' contribution for each challenge.

\subsection{Users' level of contribution behaviour}

Based on the analysis of the number of contributions posted (ideas, comments and likes) and the type of interaction (content contributions and/or simple voting), users have been clustered into different groups (Inactive; Passive; One-time contributor; Repeat contributor; Very active contributor), as shown in Table 4.3.1:

\section{User grouped by level of contribution}

\begin{tabular}{llll}
\hline & & Total & Percentage \\
\hline Inactive & no contributions and no votes & 60 & $22.6 \%$ \\
\hline Passive & no contribution, yes votes & 62 & $23.4 \%$ \\
\hline One-time contributor & 1 or 2 contributions & 40 & $16.1 \%$ \\
\hline Repeat contributor & from 3 to 10 contributions & 63 & $24.1 \%$ \\
\hline Very active contributor & up to 10 contributions & 36 & $13.8 \%$ \\
\hline \hline TOTAL & & $\mathbf{2 6 1}$ & $\mathbf{1 0 0 \%}$ \\
\hline
\end{tabular}

Table 4.3.1. Users grouped by level of contribution.

The Inactive type of user $(22.6 \%)$ is characterised by inaction: neither writing of ideas/proposals nor commenting on others' ideas nor voting activities. The Passive user (23.4\%) is characterised by some voting (others' ideas) activity but no contributions (writing ideas or commenting). About a quarter of the crowd adopted this kind of behaviour. The One-time 
contributor (16.1\%) is characterised by an exceptionally low level of activity; this type of user may have contributed to the overall discussion once or twice and represents about a quarter of the crowd. The Repeat contributor showed a moderate level of contribution behaviour (posting from three to ten ideas and/or commenting). For the contribution behaviour, this user is positioned above the One-time contributor, but below the Very active Contributor, this latter being characterised by a high level of commenting behaviour and a high level of contribution behaviour. Repeat contributor (24.1\%) and Very active Contributor (13.8\%) together represent well over one-third of the crowd.

It is also interesting to observe how these clusters changed over time. Figure 4.3.1 shows the relative size of clusters at the end of each month of the crowdsourcing event (excluding August, during which activities were suspended for summer holidays). On one hand, it is clear that the general trend does not show significant shifts. A possible explanation can be traced back to the incoming motivations and expectations: many users took part in the crowdsourcing event to obtain the professional qualification certificate, while others participated because they were interested in the topics discussed online and in the networking opportunities. Since the incoming motivations of most of the participants were sharp and clear (the attainment of the professional qualification/certificate), this may be put in relation with the decrease of inactive and passive users (the two merged categories changed from $55.3 \%$ to $46 \%$ ), as well as the increase of repeat contributors from $19.1 \%$ to $24.1 \%$, towards the end of the crowdsourcing event.

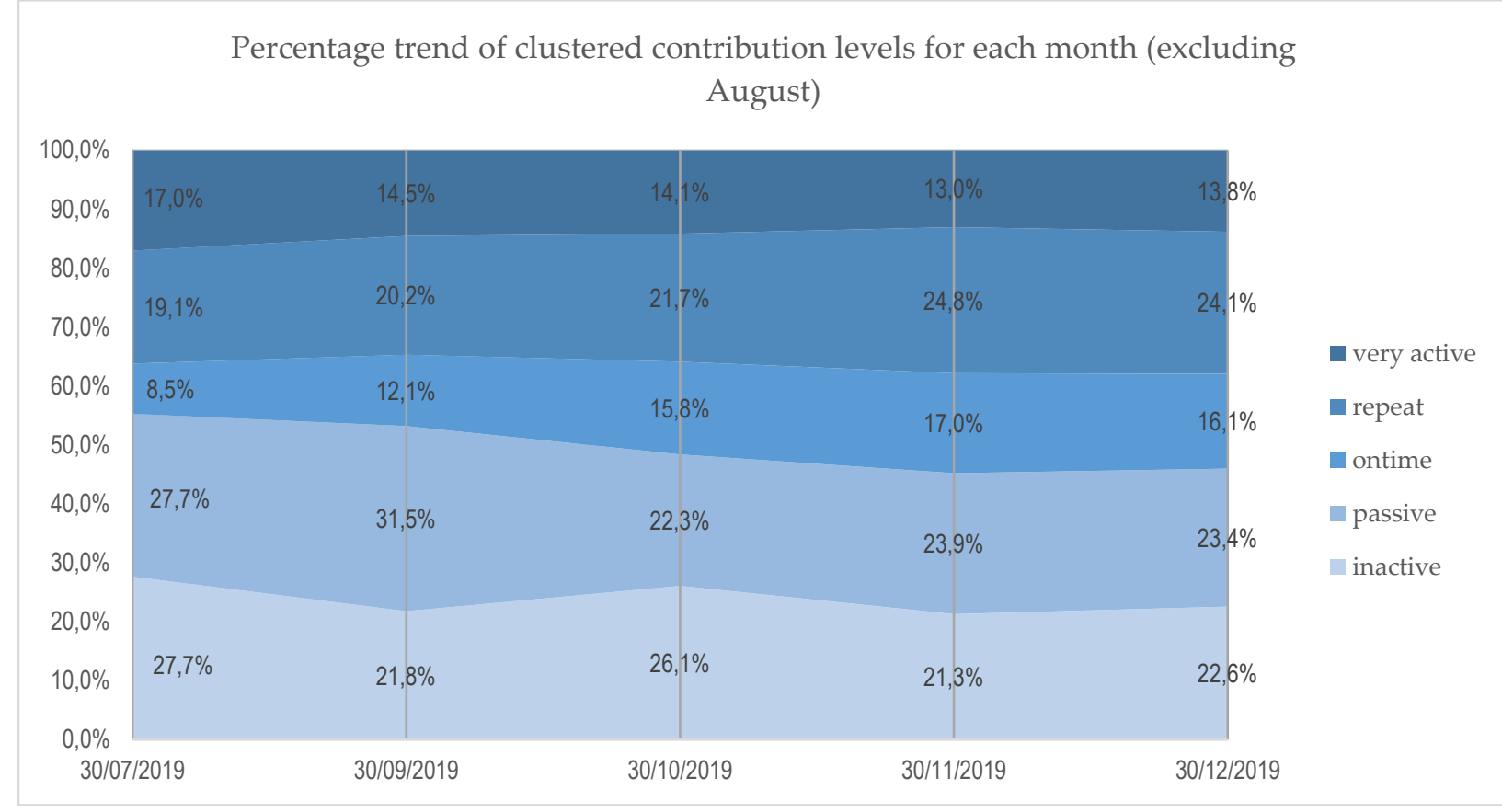

Figure 4.3.1. Trends in level of contribution during the crowdsourcing initiative Stati Generali.

\subsection{Quality of the contributions}

For the quality of the contributions, there was a wide variety of ideas and comments, some in line with the objectives of the project, others less so. We scored the quality of the contributions by levels of pertinence and relevance. Three researchers comprised the 
evaluation committee, and each evaluated all the comments and ideas posted. The researchers had to assign a score to each contribution, using a Likert scale measuring the relevance and pertinence of the contribution. The final score of each contribution was an average of the three researchers' scores.

As for the pertinence of the contributions - that is, the fact of being causally related to, and important, for the subject being considered in each challenge - we noted that only a relatively small percentage of the contributions explicitly addressed the subject of the challenge, while $75.7 \%$ did it, although only implicitly. Moreover, $13.3 \%$ of the contributions were nonpertinent and therefore unrelated to the content of the challenge discussed.

\begin{tabular}{lcc}
\hline & Frequency & Valid percentage \\
\hline Unrelated to the challenge & 118 & 13,3 \\
Implicitly addresses the challenge & 674 & 75,7 \\
Explicitly addresses the challenge & 98 & 11 \\
\hline Total & 890 & 100 \\
\hline
\end{tabular}

Table 4.4.1. Quality of the contributions: Pertinence.

Considering the relevance of the contributions, here interpreted as the degree to which a contribution is both useful and applicable to the scope of the challenge and the overall contest, in Table 4.4.2, we see that over one-third of the contributions are out of scope and only $16.2 \%$ highly relevant and therefore accurate and focused on training practices (which is the main subject of the overall contest). Even if almost half ( $47.2 \%)$ of the contributions were applicable and generalisable, suggesting a positive accomplishment of the contest, most of the overall contributions have been judged "not useful" and voted accordingly by the crowd (very useful contributions score only $1 \%$ ).

\begin{tabular}{lcc}
\hline & Frequency & Valid percentage \\
\hline $\begin{array}{l}\text { Out of scope or applicable to other areas } \\
\text { Applicable and generalizable }\end{array}$ & 326 & 36,6 \\
$\begin{array}{l}\text { With specific depth and centered on the formation (highly } \\
\text { relevant) }\end{array}$ & 420 & 47,2 \\
\hline Total & 144 & 16,2 \\
\hline
\end{tabular}

Table 4.4.2. Quality of the contributions: Relevance.

\section{Discussion}

The results presented in the previous section show that crowdsourcing makes less relevant the fact of having a community as the reference population of solvers subsequently involved in challenges and contests. Thus, the crowd dynamics (Viscusi and Tucci, 2018) plays a key role in the design of those initiatives, where it is important to distinguish between 
communities, crowds, and groups to map them into those dynamics. Indeed, it is worth noting that groups can evolve toward either communities or crowds (Viscusi and Tucci, 2018), contrary to the frequent overlapping of communities, crowds, and groups as interchangeable when used in digital environment. Then, the results have shown that the number of active contributors is lower than the one of inactive users or free riders, especially when they have a different motivation than the one defined by seeker (in our case, the certification tied to a simple participation to the contest makes less relevant providing the required policy recommendations).

However, the shadow motivation seems to reinforce the self-selection mechanisms identified as an important differentiating factor for crowdsourcing compared to communities that tends to be value intensive and inclusive when members agree on shared values (Adler, 2015; Viscusi and Tucci, 2018; West and Sims, 2018). On the learning side, the inclusion in the crowd of seeker representatives (the facilitators) is interesting, facilitating the learning dynamics that crowdsourcing may enact and the definition of the eventual "White paper" as a co-production (Cordella et al., 2018) rather than an evaluation of the winning ideas. Also, the observation about learning reported in our exploratory study is aligned with Nagle (2008: 569), who has empirically shown how organisations learn by contributing "as they receive feedback from the crowd of more experienced users and are therefore able to better capture value from using the goods". In our case, we observed a phase of stimulus where the facilitators exhibited higher expertise, followed by emergent higher expertise from self-selected members of the crowd, eventually resulting in a co-production of the final solutions. Finally, the lower degree of pertinence and relevance of the proposed solutions, on one hand, shows again a prominence of self-selection in crowdsourcing and it would require further analysis to see whether the non-pertinence/irrelevance of the ideas is contingent or else generalisable. This is a key issue for looking at the crowd capital of the contest and how many ideas could be otherwise relevant in other challenges with different goals.

Taking the above issues into account, the study could contribute to the debate on crowdsourcing by both underlining some important governance factors involved and providing empirical evidence of the link between management strategies and crowdsourcing success (in terms of the number of participants/solvers and value of the content gathered).

Uncertainty reduction theory (URT) provides a useful perspective on the interaction process between proponents (seekers) and participants (solvers), as it helps us to understand the different types of ambiguity that drive participants' behaviour as well as the role of feedback and information seeking from peers and supervisors and their relationships of with them (Callister, Kramer and Turban, 1999). Also, considering other reduction models based relational demography, uncertainty may be related to the demographic characteristics of the workgroup and its norms - for example, how to find an appropriate conversational style, or about how to behave in the workgroup; uncertainty can also be instrumental, therefore related to being unsure of what resources are needed for goal achievement (Chattopadhyay et al., 2011).

This article has the potential to contribute to further developing the distinctions between communities, crowds, and groups as emergent dynamics in crowdsourcing (challenges, topics, etc.) in their actual context. Also, we argue that our research may provide an understanding of whether and how (through which procedures, patterns, or organising forms) a social order is eventually produced in crowdsourcing. This contribution is based on looking at sense-making as a complement to the management literature that often aims to identify 
factors of individual or team performance in crowdsourcing. Finally, considering that ambiguity and uncertainty occasions sense-making at an organisational level (Weick, 1995: 91; Weick, Sutcliffe and Obstfeld, 2005), in our case those elements were strictly related to the organisational context of the challenge, both established by boundaries of institutions running the contest and emergent from the interactions of the participants to the context. Thus, we argue that URT may provide an additional complementary lens for understanding how the different actors make sense of their participation in the contest as members of a crowd, community, or a specific group and how they make use of information to make their interactions with potential strangers evolve within those forms of collectives.

Regarding limitations, our investigation, as presented in this paper, provides an exploratory view on the main issues emerging from the study of an idea contest such as the one we observed. Nevertheless, the results provide a grounded perspective on the main emerging elements worth considering in a further theoretical framework oriented to hypothesis testing. Thus, further research is in progress and we will be able to gather other data in order to test hypotheses and/or carry out other analyses. Moreover, future work will be also oriented to an investigation aimed at a more refined understanding of the "opportunism dynamics" in participation, for this scope, another component of the corpus data will be implemented with interviews with the organisers and evaluators of the contest considered in the case study as well as memos from the observation of the evaluation setting.

\section{Conclusion}

In this paper, we study two factors influencing the decision of the participants (solvers) to get involved (and to what extent) in a contest and the value perceived in participating (i.e., quality of the problem articulation, additional contextual information revealed, the status of the contest proponents). We argue that these factors affect crowdsourcing performance, measured in terms of participants' behaviour and quality of the content produced. The literature on crowdsourcing associated with Open Innovation has identified different issues worth taking into account when designing challenges and contests to support active participation and engagement; nevertheless most of the literature has focused attention on the private sector, thus, in this paper, we aim to contribute to the research on the specific patterns and mechanisms to engage an online community in crowdsourcing that could be adopted when crowdsourcing initiatives are developed in the public sector for policy or social innovation.

The analysis of the case study suggests how contest proponents (seekers) can reduce several factors of uncertainty and influence participants' decisions to get involved by means of management strategies (i.e., selecting problems where sufficient introductory knowledge is in so that knowledgeable participants can recognise and understand the challenges; revealing identity and status of the content sponsors; using members of staff as contributors at the early stage; assigning scores and prizes, etc.).

The study contributes to the debate on crowdsourcing by both underlining some important governance factors involved and providing empirical evidence of the link between management strategies and crowdsourcing success.

\section{Keywords}


crowdsourcing; crowdsourcing contest; online communities; open innovation; knowledge sharing; co-creating; crowdsourcing design and governance

\section{References}

Adler, P.S. (2015), “Community and Innovation: From Tönnies to Marx”, Organization Studies, 36 (4): 445-471. DOI: 10.1177/0170840614561566

Afuah, A., and Tucci, C.L. (2012), "Crowdsourcing as a solution to distant search", Academy of Management Review, 37 (3): 355-375. DOI: 10.5465/amr.2010.0146

Aitamurto, T., and Landemore, H. (2016), "Crowdsourced Deliberation: The Case of the Law on Off-Road Traffic in Finland", Policy \& Internet, 8 (2): 174-196. DOI: 10.1002/poi3.115

Bamberger, P.A. (2018), "AMD-Clarifying What We Are about and Where We Are Going", Academy of Management Discoveries, 4 (1): 1-10. DOI: 10.5465/amd.2018.0003

Biancani, S., McFarland, D.A., and Dahlander, L. (2014), “The Semiformal Organization”, Organization Science: 25 (5): 1306-1324. DOI: 10.1287/orsc.2013.0882

Blohm, I., Riedl, C., Leimeister, J.M., and Krcmar, H. (2011), “Idea Evaluation Mechanisms for Collective Intelligence in Open Innovation Communities: Do Traders Outperform Raters?", ICIS 2011 Proceedings, 4, 1-24.

Bogers, M., Zobel, A.K., Afuah, A., Almirall, E., Brunswicker, S., Dahlander, L., ... Ter Wal, A. L.J. (2017), "The open innovation research landscape: established perspectives and emerging themes across different levels of analysis", Industry and Innovation, 24 (1): 840. DOI: $10.1080 / 13662716.2016 .1240068$

Boudreau, K.J., and Lakhani, K.R. (2013), “Using the crowd as an innovation partner” Harvard Business Review, 91(April),

Buganza, T., and Verganti, R. (2009), "Open innovation process to inbound knowledge: Collaboration with universities in four leading firms", European Journal of Innovation Management, 12 (3), 306-325. DOI: 10.1108/14601060910974200.

Callister, R. R., Kramer, M. W., and Turban, D. B. (1999), Feedback Seeking Following Career Transitions. Academy of Management Journal, 42(4), 429-438. DOI: 10.5465/257013.

Chesbrough, H., and Bogers, M. (2014), “Explicating Open Innovation: Clarifying an Emerging Paradigm for Understanding Innovation". In H. Chesbrough, W. Vanhaverbeke, and J. West (Eds.), New Frontiers in Open Innovation, 3-28, Oxford: Oxford University Press.

Cross, R., Arena, M., Sims, J., and Uhl-Bien, M. (2017), “How to Catalyze Innovation in Your Organization", MIT Sloan Management Review, 58 (4), 39.

Dahlander, L., and Piezunka, H. (2014), "Open to suggestions: How organizations elicit suggestions through proactive and reactive attention", Research Policy, 43(5): 812-827. DOI: 10.1016/j.respol.2013.06.006.

Faraj, S., Jarvenpaa, S. L., and Majchrzak, A. (2011), “Knowledge collaboration in online communities", Organization Science, 22 (5): 1224-1239. DOI: 10.1287/orsc.1100.0614.

Faraj, S., von Krogh, G., Monteiro, E., and Lakhani, K. R. (2016), Special Section IntroductionOnline Community as Space for Knowledge Flows. Information Systems Research, 27(4), 668-684. https://doi.org/10.1287/isre.2016.0682. 
Felin, T., Lakhani, K. R., and Tushman, M. (2015), Firms, crowds, and innovation. Strategic Organization, 15(2), 119-140. DOI: 10.1177/1476127017706610.

Fleming, L., and Waguespack, D. M. (2007), “Brokerage, Boundary Spanning, and Leadership in Open Innovation Communities", Organization Science, 18 (2): 165-180. DOI: 10.1287/orsc.1060.0242

Garfinkel, H. (1967), Studies in Ethnomethodology. Reprinted 1999. Cambridge, UK: Polity Press.

Jick, T.D. (1979), "Mixing Qualitative and Quantitative Methods: Triangulation in Action”, Administrative Science Quarterly, 24 (4): 602-611.

Leimeister, J.M., Huber, M., Bretschneider, U., and Krcmar, H. (2009), "Leveraging Crowdsourcing: Activation-Supporting Components for IT-Based Ideas Competition", Journal of Management Information Systems, 26 (1): 197-224.

Linus, D., Jeppesen, L.B., and Piezunka, H. (2019), "How Organizations Manage Crowds: Define, Broadcast, Attract, and Select". In J.L. Bo, J. Sydow, and H. Berends (Eds.), Managing Inter-organizational Collaborations: Process Views (Vol. 64, 239-270), Emerald Publishing Limited. DOI: 10.1108/S0733-558X20190000064016.

Majchrzak, A., Jarvenpaa, S.L., and Bagherzadeh, M. (2015), “A Review of Interorganizational Collaboration Dynamics", Journal of Management, 41 (5): 1338-1360. DOI: $10.1177 / 0149206314563399$.

Majchrzak, A., and Malhotra, A. (2013), “Towards an information systems perspective and research agenda on crowdsourcing for innovation", The Journal of Strategic Information Systems, 22: 257-268. DOI: 10.1016/j.jsis.2013.07.004.

Majchrzak, A., and Malhotra, A. (2020), Unleashing The Crowd-Collaborative Solutions to Wicked Business and Societal Problems. Cham, Switzerland: Palgrave Macmillan/Springer Nature Switzerland AG. DOI: 10.1007/978-3-030-25557-2.

Malhotra, A., and Majchrzak, A. (2014), "Managing Crowds in Innovation Challenges", California Management Review, 56 (4): 103-123. DOI: 10.0.5.245/cmr.2014.56.4.103.

Malone, T.W. (2018), Superminds: The Surprising Power of People and Computers Thinking Together. Little, Brown.

Malone, T.W., and Bernstein, M.S. (2015), Collective Intelligence Handbook. MIT Press Cambridge, MA.

Marchegiani, L., Brunetta, F., and Annosi, M.C. (2020), "Faraway, Not So Close: The Conditions That Hindered Knowledge Sharing and Open Innovation in an Online Business Social Network". IEEE Transactions on Engineering Management, 1-17. DOI: 10.1109/TEM.2020.2983369.

O'Brien, H.L., and Toms, E.G. (2008), “What is user engagement? A conceptual framework for defining user engagement with technology", Journal of the American Society for Information Science and Technology, 59 (6): 938-955. DOI: 10.1002/asi.20801.

Olsen, W.K. (2004), “Triangulation in Social Research: Qualitative and Quantitative Methods Can Really Be Mixed". In M. Holborn and Haralambos (Eds.), Developments in Sociology. Causeway Press.

Parry, E., and Solidoro, A. (2013), "Social Media as a Mechanism for Engagement?", In Social Media in Human Resources Management (Vol. 12, 121-141), Emerald Group Publishing Limited. DOI: 10.1108/S1877-6361(2013)0000012010 
Piezunka, H., and Dahlander, L. (2018), “Idea Rejected, Tie Formed: Organizations' Feedback on Crowdsourced Ideas", Academy of Management Journal, DOI: 10.5465/amj.2016.0703.

Prpic, J., and Shukla, P. (2013), "The Theory of Crowd Capital”, 2013 46th Hawaii International Conference on System Sciences. DOI: 10.1109/HICSS.2013.545

Prpić, J., Shukla, P.P., Kietzmann, J.H., and McCarthy, I.P. (2015), “How to work a crowd: Developing crowd capital through crowdsourcing", Business Horizons, 58 (1): 77-85. DOI: 10.0.3.248/j.bushor.2014.09.005.

Puranam, P., Alexy, O., and Reitzig, M. (2014), “What's 'new' about new forms of organizing?" Academy of Management Review, 39 (2): 162-180. DOI: 10.5465/amr.2011.0436.

Retelny, D., Robaszkiewicz, S., To, A., Lasecki, W.S., Patel, J., Rahmati, N., ... Bernstein, M.S. (2014), "Expert Crowdsourcing with Flash Teams". In Proceedings of the 27th Annual ACM Symposium on User Interface Software and Technology, 75-85, New York, NY, USA: ACM. DOI: $10.1145 / 2642918.2647409$

Robinson, S.L. (2019), “What Is a Pre-Theory Paper? Some Insights to Help You Recognize or Create a Pre-Theory Paper for AMD", Academy of Management Discoveries, 5 (1): 1-7. DOI: 10.5465/amd.2019.0059.

Rowe, G., and Frewer, L.J. (2005), "A Typology of Public Engagement Mechanisms", Science, Technology, \& Human Values, 30 (2): 251-290. DOI: 10.1177/0162243904271724.

Schaufeli, W.B. (2014), “What is engagement?" In C. Truss, K. Alfes, R. Delbridge, A. Shantz, and E. Soane (Eds.), Employee engagement in theory and practice, 15-35, Abingdon, Oxon, UK: Routledge.

Seidel, V.P., Langner, B., and Sims, J. (2016), “Dominant communities and dominant designs: Community-based innovation in the context of the technology life cycle", Strategic Organization, 15 (2): 220-241. DOI: 10.1177/1476127016653726.

Sims, J., and Woodard, C. J. (2019), "Community interactions at crowd scale: hybrid crowds on the GitHub platform". Innovation: 1-23. DOI: 10.1080/14479338.2019.1585860.

Siobhan, O. (2011), “Organizations in the Shadow of Communities". In L.K.R., C. Marquis, M. Lounsbury, and R. Greenwood (Eds.), Communities and Organizations, Vol. 33, 3-36, Emerald Group Publishing Limited. DOI: 10.1108/S0733-558X(2011)0000033004.

Stieger, D., Matzler, K., Chatterjee, S., and Ladstaetter-Fussenegger, F. (2012), “Democratizing Strategy: How Crowdsourcing Can be Used for Strategy Dialogues", California Management Review, 54 (4): 44-68. DOI: 10.1525/cmr.2012.54.4.44.

Storbacka, K., Brodie, R.J., Böhmann, T., Maglio, P.P., and Nenonen, S. (2016), “Actor engagement as a microfoundation for value co-creation", Journal of Business Research, 69 (8): 3008-3017. DOI: 10.1016/j.jbusres.2016.02.034.

Tucci, C.L., Afuah, A., and Viscusi, G. (2018), Creating and Capturing Value Through Crowdsourcing. Oxford University Press.

Tucci, Christopher, Viscusi, G., and Gasparetto, F. (2016), “Distinguishing Crowd Dynamics in Small Teams: A Crowdsourcing Exercise in Higher Education". In Collective Intelligence 2016. New York, NY, USA. Retrieved from https://infoscience.epfl.ch/record/218646/files/CI-TUVIGA_vPRINT.pdf.

Unimib.crowdicity.com. (2020), Stati Generali della Formazione e del Lavoro. Retrieved December 2, from https://unimib.crowdicity.com/. 
Valentine, M.A., and Edmondson, A.C. (2014), "Team Scaffolds: How Mesolevel Structures Enable Role-Based Coordination in Temporary Groups", Organization Science, 26 (2): 405-422. DOI: 10.1287/orsc.2014.0947.

Valentine, M.A., Retelny, D., To, A., Rahmati, N., Doshi, T., and Bernstein, M.S. (2017), "Flash Organizations: Crowdsourcing Complex Work by Structuring Crowds As Organizations". In Proceedings of the 2017 CHI Conference on Human Factors in Computing Systems (pp. 3523-3537), New York, NY, USA: Association for Computing Machinery. DOI: $10.1145 / 3025453.3025811$.

Viscusi, G., and Tucci, C. (2018), “Three's a Crowd?”. In Christopher Tucci, A. Afuah, and G. Viscusi (eds.), Creating and Capturing Value through Crowdsourcing. Oxford University Press.

Weick, K.E. (1995), Sensemaking in organizations. Thousand Oaks, CA: SAGE Publications Inc.

Weick, K.E., Sutcliffe, K.M., and Obstfeld, D. (2005), “Organizing and the Process of Sensemaking", Organization Science, 16 (4): 409-421. DOI: 10.1287/orsc.1050.0133

West, J., and Sims, J. (2018), How Firms Leverage Crowds and Communities for Open Innovation. In C. Tucci, A. Afuah, and G. Viscusi (eds.), Creating and Capturing Value through Crowdsourcing, 58-96, Oxford, UK: Oxford University Press. https://doi.org/10.1093/oso/9780198816225.001.0001. 